AperTO - Archivio Istituzionale Open Access dell'Università di Torino

Hyaluronic acid-conjugated lipoplexes for targeted delivery of siRNA in a murine metastatic lung cancer model.

This is a pre print version of the following article:

Original Citation:

Availability:

This version is available http://hdl.handle.net/2318/1615996

since 2018-12-07T14:24:44Z

Published version:

DOI:10.1016/j.ijpharm.2016.06.125

Terms of use:

Open Access

Anyone can freely access the full text of works made available as "Open Access". Works made available under a Creative Commons license can be used according to the terms and conditions of said license. Use of all other works requires consent of the right holder (author or publisher) if not exempted from copyright protection by the applicable law. 


\section{Hyaluronic acid-conjugated lipoplexes for targeted delivery of siRNA in a murine metastatic lung cancer model}

Thais Leite Nascimento ${ }^{\mathrm{a}, \mathrm{b}, \mathrm{c}}$, Hervé Hillaireaua, ${ }^{\mathrm{b}}$, Juliette Vergnauda, ${ }^{\mathrm{b}}$, Melania Rivanoa, ${ }^{\mathrm{b}}$, Claudine Deloménie $^{\mathrm{d}}$, Delphine Courilleau ${ }^{\mathrm{d}}$, Silvia Arpicco ${ }^{\mathrm{e}}$, Jung Soo Sukf,,’h, Justin Hanesf,g,h, Elias Fattal ${ }^{\mathrm{a}}, \mathrm{b}, *$

a Université Paris-Sud, Faculté de Pharmacie, Institut Galien Paris-Sud, 5 rue JB Clément, 92296 Châtenay-Malabry Cedex, France

b CNRS, UMR 8612, 5 rue JB Clément, 92296 Châtenay-Malabry Cedex, France

c CAPES Foundation, Ministry of Education of Brazil, Brasília___DF 70040-020, Brazil

d UMS-IPSIT-US 31 Inserm-UMS 3979 CNRS, 5 rue JB Clément, 92296 Châtenay-Malabry Cedex, France

e Università degli Studi di Torino, Facoltà di Farmacia, Dipartemento di Scienza e Tecnologia del Farmaco, Via Pietro Giuria 9, 10125 Torino, Italy

f Center for Nanomedicine, Wilmer Eye Institute, Johns Hopkins University School of Medicine, Baltimore, MD 21231; USA, USA

g Department of Ophthalmology, Wilmer Eye Institute, Johns Hopkins University School of Medicine, Baltimore, MD 21297; USA, USA

h Department of Chemical and Biomolecular Engineering, Johns Hopkins University, Baltimore, MD 


\begin{abstract}
We have investigated the impact of hyaluronic acid (HA)-coating on the targeting capacity of siRNA lipoplexes to CD44-overexpressing tumor cells. Cellular uptake and localization of HA-lipoplexes were evaluated by flow cytometry and fluorescence microscopy and both methods showed that these lipoplexes were rapidly internalized and localized primarily within the cytoplasm. Inhibition of luciferase expression on the A549-luciferase lung cancer cell line was achieved in vitro using an antiLuc siRNA. $81 \%$ of luciferase gene expression inhibition was obtained in vitro with HA-lipoplexes at $+/$ _ ratio 2. In vivo, in a murine A549 metastatic lung cancer model, the treatment with HAlipoplexes carrying anti-luciferase siRNA led to a statistically significant decrease of luciferase expression as opposed to progressive increase with non-modified lipoplexes or $\mathrm{NaCl} 0.9 \%$. The reduction of the expression of luciferase mRNA tumor of mice treated with HA-lipoplexes supported the inhibition effect due to siRNA. These results highlight the potential of HA-lipoplexes in CD44targeting siRNA delivery.
\end{abstract}

Keywords: Lipoplexes siRNA Hyaluronic acid A549 CD44 Luciferase 


\section{Introduction}

Downregulation of gene expression is a promising strategy that meets different applications in therapeutics. Small interfering RNA (siRNA) molecules present the inherent advantage of nucleic acid therapies consisting in the almost unrestricted choice of targets (Aagaard and Rossi, 2007). SiRNA has shown potentialities for the treatment of lung diseases including the treatment of inflamma-tory, immune and infectious diseases, cystic fibrosis (CF) and cancer (Nascimento et al., 2012). However, their clinical use, even for lung diseases, is still limited due to the same obstacles faced by other nucleotide-based therapeutics. Indeed, siRNA are rapidly degraded by nucleases showing half-lives in biological fluids of the order of seconds to minutes (Soutschek et al., 2004). Moreover, siRNA lacks selectivity for the targeted tissue (Aagaard and Rossi, 2007; Devi, 2006; Fattal and Barratt, 2009; Fattal and Bochot, 2008; Nascimento et al., 2012). Within the tissues, they do not cross cell membranes readily because of their negative charge, hydrophilicity and molecular size (Dykxhoorn and Lieberman, 2006; Fattal and Barratt, 2009). To overcome these limitations and enable siRNA delivery to their site of action, different nanocarriers systems have been investigated, including the biocompatible lipid-based liposomes, or lipoplexes. It was shown that surface modification of liposomes with high molecular weight hyaluronic acid (HA) can improve their efficacy by mediating active CD44 targeting in tumors (Arpicco et al., 2013b; Dalla Pozza et al., 2013; Glucksam-Galnoy et al., 2012; Landesman-Milo et al., 2013; Peer and Margalit, 2004a,b; Rivkin et al., 2010; Ruhela et al., 2014; Yang et al., 2013). HA is a glycosaminoglycan polymer composed of disaccharide units of $\mathrm{N}$-acetylglucosamine and $\mathrm{D}$-glucuronic acid linked together through alternating b-1,3 and b-1,4 glycosidic * Corresponding author at: Université Paris_Sud, Faculté de Pharmacie, Institut Galien Paris_Sud, UMR CNRS 8612, 5 rue JB Clément, 92296 Châtenay _Malabry Cedex, France. E-mail address: elias.fattal@u-psud.fr (E. Fattal). http://dx.doi.org/10.1016/j.ijpharm.2016.06.125 0378-5173/ã_2016 Elsevier B.V. All rights reserved. International Journal of Pharmaceutics 514 (2016) 103-111 Contents lists available at ScienceDirect International Journal of Pharmaceutics journal homepage: www.elsev ier.com/locate / ijpharmbonds. It is biocompatible, being the major component of the extracellular matrix. The native high molecular weight HA is non-toxic and non-immunogenic (Laurent and Fraser, 1992). It does not induce expression of genes involved in proliferation or inflamma-tion (Noble, 2002) and counteracts proangiogenic effects of the HA oligomers (Deed et al.,1997; Dufay Wojcicki et al., 2012). At last but not least, HA can be utilized as an addressing molecule due to the expression of its membrane receptor, CD44, on tumor initiating cells (Al-Hajj et al., 2003) that are the main cells to target in order to avoid tumor relapse. Indeed, although some studies have focused on targeting CD44 using antibodies (Wang et al., 2012) or aptamers (Alshaer et al., 2015), most of them were related to the 
use of HA (Dosio et al., 2016). HA can also increase liposome circulation time due to possible dysopsonisation effects (Peer et al., 2003; Peer and Margalit, 2004b; Qhattal and Liu, 2011). Moreover, several groups have shown that it is possible to mediate siRNA delivery by nanotechnologies covered by HA such as polyethyle- nimine (Ganesh et al., 2013) or lipid particles (Landesman-Milo et al., 2013). For several years we have studied the effects of surface coverage of lipoplexes by HA through a HA-DOPE conjugate that is inserted within the lipoplex structure. The molecular organization of these lipoplexes was recently characterized (Dufay Wojcicki et al., 2012; Nascimento et al., 2015; Surace et al., 2009; Taetz et al., 2009). An improved and receptor-mediated transfection efficiency of breast and lung cancer cells overexpressing CD44 receptors was reported (Dufay Wojcicki et al., 2012; Surace et al., 2009). In a preliminary study, it was also shown that siRNA lipoplexes covered with HA could enter at a larger extent into A549 CD44+ cells than Calu3 CD44_cells (Taetz et al., 2009). However this last study did not demonstrate any inhibition effect provided by the siRNA. This is the reason why the aim of the present report was to provide evidences of success of such a strategy. HA-DOPE modified cationic siRNA lipoplexes were designed using a non-commercialized cationic lipid that has demonstrated promising transfection efficiency in different cell lines (Arpicco et al., 2004; De Rosa et al., 2008), the [2-(23 didodecyloxypropyl)hydroxyethyl] am-monium bromide (DE). The effects of this modification on cell internalization were evaluated. Also, the ability of the lipoplexes to carry intact siRNA to the cytoplasm was assessed by testing gene expression inhibition on the A549-luc lung cancer cell line in vitro and for the first time in an in vivo lung cancer experimental model.

\section{Materials and methods}

\subsection{Materials}

The cationic lipid [2-(2-3didodecyloxypropyl)hydroxyethyl] ammonium bromide (DE) was synthesized as described (Arpicco et al., 2004). L-alpha-dioleylphosphatidylethanolamine (DOPE) and phosphatidylethanolamine conjugated to rhodamine (PE-rhodamine) were purchased from Avanti Polar Lipids distributed by Sigma Aldrich (Saint Quentin Fallavier, France). High molecular weight hyaluronic acid (HA) (sodium salt, $1600 \mathrm{kDa}$, purity of 95\%) was provided by Acros organics (Geel, Belgium). pGL3 luciferase (firefly) and control siRNA (19 bp) were purchased from Euro-

gentec (Angers, France). The HA-DOPE conjugate was synthesized as described previously (Surace et al., 2009).

\subsection{Liposomes and lipoplexes preparation and characterization}

Liposomes of DOPE/DE at 1:1 w/w ratio were prepared in water by the ethanol injection method (Batzri and Korn, 1973; Taetz et al., 2009). The preparation protocol is described in the 
supplementary information. The HA-DOPE content of liposomes is expressed as mass ratio of HADOPE to other lipids (DE + DOPE) $(10 \%$ refers to 1:10 w/w). Lipoplexes were prepared at charge ratios (+/ _ ratios) of 2 and 134 by adding one volume of the $3 \mathrm{mM}$ liposome suspension into two volumes of siRNA solution at 11.05 or $0.16 \mathrm{mM}$, respectively, in an Eppendorf tube, and gently homogenizing by pipetting up and down. The ratios were calculated based on the fact that one positive charge is provided by $1 \mathrm{~mol}$ of DE and 38 negative charges are brought by 1 mol of siRNA. Suspensions of $15 \mathrm{~mL} \_2.5 \mathrm{~mL}$ of lipoplexes were usually prepared and incubated for $1 \mathrm{~h}$ at room temperature before use. Lipoplexes diameter, zeta potential and stability in the presence of serum were characterized as described in the supplementary information.

\subsection{Cell culture}

A549-luc-C8 Bioware Cell Line, a luciferase-expressing cell line derived from A549 adenocarcinomic human alveolar epithelial cells, was purchased from Caliper Life Sciences (Hopkinton, USA). Cells were cultured using RPMI-1640 medium supplemented with 10\% FBS, 50 units $/ \mathrm{mL}$ penicillin and 50 units/mL streptomycin. They were maintained at $37^{\circ} \mathrm{C}$ in a humidified atmosphere with 5\% CO2. To improve the homogeneity of luciferase expression and increase luminescence signals, a protocol for selection pressure was optimized. Before each experiment, cells were cultured for 12 days using the RPMI medium described previously with addition of $75 \mathrm{mg} / \mathrm{ml}$ Geneticin1G418 antibiotic (Gibco, Paisley, Scotland). CD44 expression on A549, A549-luc and G418-selected A549-luc cells was evaluated by flow cytometry (method described in the supplementary Information). Almost $100 \%$ of the cells expressed CD44 receptors on their surface, and the amount of receptors did not change after treatment with G418 (Table S1 and Fig. S1, Supplementary Information).

\subsection{Cell viability}

Cellular mitochondrial activity was evaluated after incubation with liposomes and lipoplexes using the 3-[4,5-dimethylthiazol-2-yl]-3,5-diphenyl tetrazolium bromide (MTT) test. Cells were seeded in 96-well plates at a density of 70,000 cells $/ \mathrm{mL}$ and allowed to adhere. After $24 \mathrm{~h}$, cells were rinsed with PBS and fresh serum-free medium was added to the wells. Liposomes and lipoplexes were diluted in RPMI serum-free medium and added to the wells at various lipid concentrations (0.3-272 $\mathrm{mM}$ ). Six hours after incubation, serum was added to the wells at $10 \% \mathrm{v} / \mathrm{v}$, and cells were incubated for $48 \mathrm{~h}$. Then, $20 \mathrm{~mL}$ of a $5 \mathrm{mg} / \mathrm{mL}$ MTT solution was added to each well. After $2 \mathrm{~h}$ of incubation at $37^{\circ}$ C , the medium was discarded and $100 \mathrm{~mL}$ of DMSO was added to lyse the cells and solubilize the formazan crystals. The absorbance was measured with a micro-plate reader at $540 \mathrm{~nm}$. Each liposome or lipoplex concentration was evaluated in triplicate, and the experiment was performed at 
least three times. Cell viability was expressed as the percentage of mitochondrial activity relative to the non-treated cells.

\subsection{Lipoplexes uptake}

Cells were seeded on 12-well plates at a density of 72,000 cells/ $\mathrm{mL}$ and allowed to adhere. After 24 $\mathrm{h}$, cells were rinsed with PBS, and fresh serum-free medium was added to the wells. Rhodaminelabeled liposomes and lipoplexes at +/ _ ratios 2 and 134 were diluted in RPMI serum-free medium and added into the wells at a final lipid concentration of $10 \mathrm{mM}$ (67 nM of siRNA for lipoplexes at +/_ ratio 2 and $1 \mathrm{nM}$ of siRNA for lipoplexes 134), and incubated at $37^{\circ}$ C. After 2, 5, 24 or $48 \mathrm{~h}$, supernatants were discarded, and cells rinsed twice with PBS and harvested by 1 _ trypsine. This step was introduced to ensure that particles attached to the surface of cells, but not internalized, would not be taken into account in the flow cytometry measurements, possibly giving false-positive results. Cell suspensions were analyzed by flow cytometry, and mean fluorescence intensities (MFI) were collected on channel FL-2. Results were expressed as the ratio of the MFI of each sample to the MFI of non-treated cells. All measurements were performed in triplicate. In these experiments the term uptake was considered as the sum of particle membrane associated and internalized.

\subsection{Subcellular localization of lipoplexes}

The intracellular trafficking of lipoplexes +/_ ratios 2 and 134 was investigated on A549 cells using confocal laser scanning microscopy. A549 cells were seeded at a density of 150,000 cells/ $\mathrm{mL}$ on coverslips and allowed to adhere at $37^{\circ} \mathrm{C}, 5 \% \mathrm{CO}$. After $24 \mathrm{~h}$, cells were rinsed with PBS and fresh serum-free medium was added to the wells. Cells were then treated with rhodamine- labeled lipoplexes at a final lipid concentration of $10 \mathrm{mM}$. After $2 \mathrm{~h}, 6 \mathrm{~h}$ or $24 \mathrm{~h}$ of incubation, cells were rinsed with PBS and fixed by addition of $800 \mathrm{~mL}$ of $4 \%$ paraformaldehyde in PBS (w/v) during 20 min. After washing with PBS, $10 \mathrm{~mL}$ of Vectashield1 mounting medium for fluorescence (Vector Laboratories, Inc., Burlingame, USA) was used to prepare the slides. Fluorescence experiments were performed with a confocal laser scanning microscope LSM 510-Meta (Zeiss, Germany) using a 63 _ /1.4 plan-Apochromat objective lens, a helium neon laser (excitation wavelength $543 \mathrm{~nm}$ ) and a long pass emission filter LP $560 \mathrm{~nm}$. The pinhole was set at 1.0 Airy unit (0.8 mm optical slice thickness). Twelve bit numerical images were acquired with LSM 510 software version 3.2.

\subsection{In vitro luciferase inhibition}

The ability of lipoplexes to inhibit luciferase gene expression on A549-luc cells was carried out. For that, cells were seeded on 96- well plates at a density of 30,000 cells $/ \mathrm{mL}$ and allowed to adhere. After $24 \mathrm{~h}$, cells were rinsed with PBS, and fresh serum-free medium without red phenol was added to the wells. Lipoplexes were prepared with control and anti-luciferase siRNA at +/ _ ratios 134 and 2, with non-modified or HA-liposomes. Lipoplexes were diluted in serum-free medium and added into the 
wells at a final lipid concentration of $100 \mathrm{mM}$. Six hours after incubation, serum was added to the wells at $10 \% \mathrm{v} / \mathrm{v}$ and cells were incubated for $48 \mathrm{~h}$. Fifty microliters of BriteLite (Perkin-Elmer Life Sciences, Courta-boeuf, France), containing both lysis buffer and luciferin substrate were then added to the wells. Plates were vortexed for $3 \mathrm{~min}$ and luciferase enzyme activity was quantified using the Envision Xcite luminometer (Perkin ElmerLife Sciences, Courtaboeuf, France) at ultrasensible mode, with measurement time of $0,1 \mathrm{~s} /$ well. Meas-urements were performed in 8 replicates for each sample of lipoplexes. Luciferase activity of untreated cells was measured simultaneously as a baseline for comparison. Controls of non-targeting siRNA lipoplexes were used to observe the effect of the formulations on luciferase activity.

\subsection{Distribution of lipoplexes in mouse lung}

Animal studies were carried out in strict accordance to the recommendations in the Guide for the Care and Use of Laboratory Animals of the National Institutes of Health. To investigate the distribution of lipoplexes in mouse lungs, healthy CF-1 mice (female, 6-8 weeks) were treated with a single i.v. injection of rhodamine-labeled non-modified or HA-lipoplexes containing $20 \mathrm{mg}$ of siRNA. Thirty minutes later, mice were euthanized using an excess of isoflurane. Lungs were dissected and flashfrozen with liquid nitrogen in Tissue-Tek O.C.T. Compound. Transverse sections of $10 \mathrm{~mm}$ of thickness were obtained at various points along the length of the tissue using a Leica CM-3050-S cryostat. The sections were stained with ProLong1 Gold antifade reagent with DAPI (Molecular Probes, Eugene, OR) and images were captured with an inverted epifluorescence microscope (Zeiss Axio Observer).

\subsection{A549 intravenous experimental lung metastatic model}

Experiments were conducted according to the European rules (86/609/EEC and 2010/63/EU) and the principles of laboratory animal care and legislation in force in France (Decree No. 2013-118 of February 1, 2013). Five-week-old female athymic nude mice (Foxn1nu) were purchased from Harlan Laboratories (Gannat, France) and maintained in specific pathogen-free conditions throughout the experiment. Animals were kept in sterile cages (maximum of four mice/cage) bedded with sterilized soft wood granulate at a $12 \mathrm{~h} \mathrm{light/dark} \mathrm{cycle,} \mathrm{and} \mathrm{supplied} \mathrm{with} \mathrm{chow} \mathrm{and} \mathrm{autoclaved} \mathrm{water.} \mathrm{All}$ manipulations were performed under a laminar flow hood. A459-luc cells at $80 \%$ confluence were harvested, washed 3 times and suspended in PBS. Mice were injected in the tail vein with 1.5 million cells suspended in $200 \mathrm{~mL}$ of PBS, and tumor growth in the lungs was monitored weekly by bioluminescence imaging. Bioluminescence from luciferase expressing A549 cells (A549- luc) was measured using IVIS1 Lumina Series III LT (Perkin Elmer, USA). Firefly luciferin (Perkin Elmer, USA) was injected intraperi- toneally $6 \mathrm{~min}$ before imaging at a concentration of $100 \mathrm{mg} / \mathrm{kg}$. Mice were then anesthetized using $4 \%$ isoflurane and placed on a warmed stage inside the light-tight 
camera box with continuous exposure to $2.5 \%$ isoflurane. Images were made from ventral and dorsal views from 6 to 15 min after luciferin injection. Data were analyzed based on total photon emission (photons/sec) in the region of interest over the thoracic space.

\subsection{In vivo luciferase inhibition}

The in vivo delivery of luciferase siRNA was tested in female athymic nude mice bearing the A549luc metastatic cancer. Bioluminescence measurements were made $24 \mathrm{~h}$ before treatment. Each group ( 8 animals) was then treated once a day for 3 days by i.v. injection of either $\mathrm{NaCl} 0.9 \%$, luciferasesiRNA solution, HA-lipoplexes prepared with nonspecific control siRNA, HA-lipoplexes prepared with luciferase siRNA or non-modified lipoplexes prepared with luciferase siRNA. Total siRNA amount injected was $60 \mathrm{mg} / \mathrm{mouse}$. At $24 \mathrm{~h}$ post treatment, bioluminescence was quantified, mice were euthanized and lungs were removed for siRNA quantification by qPCR. Efficiency of gene expression inhibition in vivo was quantified in each mouse by the ratio between luminescence immediately before and $24 \mathrm{~h}$ after the last treatment. The lungs from 4 siRNA lipoplex-treated and 3 vehicle-treated mice were frozen in situ with liquid nitrogen, and tissue was stored at $\_80 \_\mathrm{C}$ until submitted to RNA extraction. The whole lung tissue from each animal was homogenized with ceramic beads in a Precellys 24 (Bertin, France) before extraction of total RNA using Trizol RNA isolation reagent (Life Technologies, France) according to the manufacturer's instructions. RNA purity and quantity was assessed by UV measurement using a BioMate $3 \mathrm{~S}$ spectrophotom- eter (Thermo Scientific, France). RNA integrity was evaluated by capillary electrophoresis using RNA 6000 Nano chips and the Bioanalyzer 2100 (Agilent Technologies, USA). For quantification of mRNA expression, strand cDNAs were first reverse-transcribed from $1 \mathrm{mg}$ of total RNA, with random hexamers and oligo-dT priming using the iSCRIPT enzyme (Bio-Rad Laboratories, USA), according to the manufacturer's instructions. PCR primer pairs specific to the target and reference genes (Table 2, Supplementary Information) were designed using Primer3Plus software. cDNAs synthesized from $4 \mathrm{ng}$ of total RNAs were amplified in a CFX96 real time thermal cycler (Bio-Rad) using the SSoADV Univer SYBR Green Supermix (Bio-Rad) reagent, with $500 \mathrm{nM}$ final concentrations of each primer, in duplicates of $10 \mathrm{~mL}$ reactions, by 45 two-step cycles (95_C $5 \mathrm{~s}$; 60 _C 30 s). 'No RT' _controls were amplified on all genes to verify genomic DNA contamination, and melting curve analysis was performed to assess the purity of the PCR products. PCR efficiencies, calculated for each gene from the slopes of calibration curves generated from the pool of all cDNA samples, were above 95\%. GeNorm in qBase Plus tool (Vandesompele et al., 2002) was used to select r18S, HPRT, TBP and ACTB genes as references for normalization of mRNA expression results. The normalized relative expression of target genes in samples was determined using the DDCq method with correction for PCR efficiencies, where NRQ = ETarget _ DCqTarget/ERef _ DCqRef and DCq 
$=$ Cqsample ${ }_{-}$Cqcalibrator (Hellemans et al., 2007). Final results were expressed as the n-fold differences in target gene expression in lipoplex-treated vs vehicle-treated mice.

\subsection{Statistical analysis}

Statistical analysis of data was performed by analysis of variance (ANOVA) followed by Bonferroni or Mann-Whitney's (for qPCR) tests. Differences were considered to be statistically significant at a level of $\mathrm{P}<0.05 .3$.

\section{Results}

\subsection{Lipoplexes preparation and stability}

The mean diameter, polydispersity index (PdI), zeta potential and amount of siRNA associated to the obtained non-modified and HA-modified liposomes and lipoplexes are reported in Table 1. Insertion of the HA-DOPE conjugate in lipoplex structure caused the increase in the mean diameter, from 76

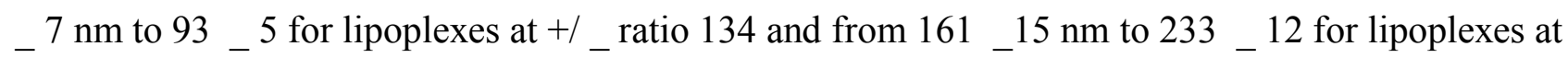
$+/$ _ ratio 2. The decrease in the surface charge upon addition of siRNA and formation of lipoplexes confirmed the association of the densely charged siRNA molecules. When non-modified and HAliposomes or lipoplexes were diluted in cell culture medium, a difference in their diameter was observed (Fig. S2). HA-liposomes and lipoplexes had a lesser tendency to aggregate in the presence of the serum present in the medium compared to their non-modified counterparts which display a significant increase in their diameter due to aggregation-mediated by serum proteins (Fig. S2).

\subsection{Cytotoxicity}

As a first step for in vitro experiments, a comparative cytotoxicity test was performed on A549-luc cells using non-modified and HA-modified liposomes and lipoplexes at +/_ ratios of 2 and 134. Cells were incubated for $48 \mathrm{~h}$ with liposomes/ lipoplexes formulations at concentrations ranging from 0.1 to $300 \mathrm{mM}$ lipids. The results show that the modification by HA and the presence of siRNA in the samples did not alter their toxicity profiles (Fig. 1). To ensure a cell viability above $80 \%$, the lipid concentration was maintained at $10 \mathrm{mM}$ for further in vitro experiments.

\subsection{Lipoplexes uptake and intracellular distribution}

The uptake of liposomes/lipoplexes by A549-luc cells was investigated using fluorescently labeled, non-modified and HA-modified liposomes and lipoplexes at at + / $_{-}$ratios of 134 and 2 . The uptake was quantified by flow cytometry after the cell-associated fluorescence was eliminated by rinsing cells twice with PBS and harvesting by trypsine (Fig. 2). A progressively increasing uptake from 2 to $48 \mathrm{~h}$ was observed for liposomes and lipoplexes (ratio $\left.+/{ }_{-} 134\right)$. At all analyzed times, uptake of liposomes containing the HA-DOPE conjugate was higher compared to the non-modified particles. The same trend was observed for lipoplexes at a ratio of $+{ }_{-}{ }_{134}$. HA-lipoplexes at a $+/$ ratio of 2 
were internalized more rapidly compared to all other analyzed particles. At $2 \mathrm{~h}$, a plateau of the uptake of these lipoplexes was already observed, and high cellular fluorescence intensity was observed until $48 \mathrm{~h}$ with however no differences between modified and non-modified particles. The intracellular distribution of lipoplexes in A549-luc cells was assessed by confocal laser microscopy using nonmodified and HA-rhodamine-labeled lipoplexes at +/_ ratios 2 Table 1 Mean diameter, polydispersity index (PdI), zeta potential and \% of siRNA associated of non-modified and HA-liposomes and lipoplexes prepared at ratios $+/ \ldots 134$ and $2(\mathrm{n}=3)$. Composition Diameter $(\mathrm{nm})$ PdI Zeta Potential $(\mathrm{mV}) \%$ siRNA associated DE:DOPE 90 _ 120.243 _ 0.0657 _ 2 - DE:DOPE:DOPE-HA 108 _ 30.216 _ 0.0435 _ 0.8 _ _DE:DOPE:siRNA (134) 76 _ 70.268 _ 0.0446 _ 1.399 .2 _ 0.7 DE:DOPE:DOPE-HA:siRNA (134) 93 _ 50.307 _ $0.0230 \_1.799 .0$ _ 0.4 DE:DOPE:siRNA (2) 161 _ $150.171 \_0.0628$ _ $1.993 .0 \_0.6$ DE:DOPE:DOPE-HA:siRNA (2) 233 _ 120.151 _ 0.03 _ 42 _ 396.0 _ 2.6 Fig. 1. A549-luc mitochondrial activity as a function of lipid concentration, determined by MTT test after incubation with liposomes and lipoplexes. Cells were incubated for 48 $\mathrm{h}$ with non-modified and HA-liposomes and lipoplexes at $+{ }_{-}$ratios 2 and 134, at concentrations from 0.1 to $300 \mathrm{mM}$ lipids/well $(\mathrm{n}=3) .106$ with fluorescently-labeled siRNA. The diffuse fluorescence ob-served in ortho-images confirmed lipoplex (lipids and siRNA) localization in the cell cytoplasm, rather than merely bound to the cell membrane. Images from 2, 6 and $24 \mathrm{~h}$ after incubation (Fig. S3) show a progressive internalization of lipoplexes, as observed by the augmentation of global lipid fluorescence from 2 to $6 \mathrm{~h}$, after which the fluorescence plateaued.

\subsection{In vitro luciferase gene expression inhibition}

The ability of lipoplexes to carry siRNA into the cell cytoplasm was then evaluated by measuring the inhibition of luciferase activity in A549-luc cells. Differences between non-modified and HA-DOPE lipoplexes were studied with lipoplexes at + / $_{\text {_ }}$ ratios 134 and 2 prepared using luciferase-specific siRNA and non-specific control siRNA. The results in Fig. 3 show that specific inhibition by luciferase siRNA was observed compared to control (scramble) siRNA. For a + /_ ratio 134, ca. 63\% luciferase inhibition was observed after incubation with lipoplexes modified by the HA-DOPE conjugate, compared to ca. $23 \%$ for the non-modified lipoplexes. The highest luciferase inhibitions were obtained with lipoplexes at $+/$ _ ratio 2 , with ca. $70 \%$ for the non-modified and ca. $81 \%$ for the HA-modified lipoplexes. For this reason and for the possibility to administer higher doses, lipoplexes at $+/$ _ ratio 2, HA-modified or not, were used in further in vivo experiments.

\subsection{Lung distribution of lipoplexes}

To visualize the influence of HA surface modification of lipoplexes on their lung distribution following intravenous administration to mice, fluorescently labeled lipoplexes were prepared at + / ratio 2, HA-modified or not. After i.v. injection to CF mice, particle distribution was observed using 
fluorescence microscopy. Fig. 4 shows representative images of the lipoplexes distribution in the lungs. A large difference in tissue distribution was observed between these two types of particles 30 min following administration. The fluorescence related to HA-lip-oplexes was homogenously distributed throughout the lungs. Meanwhile, weak fluorescence spots were observed for the nonmodified lipoplexes, evidencing the sparse distribution and the formation of aggregates.

\subsection{Tumor growth and in vivo luciferase inhibition}

To test the ability of HA-lipoplexes to silence gene expression in vivo, we developed a metastatic tumor model using the luciferase- expressing A549-luc cells. 1.5 million cells suspended in $200 \mathrm{~mL}$ of PBS were injected in the tail vein of athymic nude mice, and tumor growth was monitored weekly by bioluminescence imaging. A steady progression of lung tumor growth was measured over the period of 40 days, which correlated to the finding of tumor nodules on the macroscopic analysis of lungs. No changes in the body weight of mice were observed throughout the study. Fig. S4 shows tumor growth measured by BLI imaging, typical time-dependent ventral images of a mouse with average bioluminescence demonstrating lung colonization over time, and a representative image of lungs dissected 40 days after cell injection. The tumor targeting potential and the ability of lipoplex

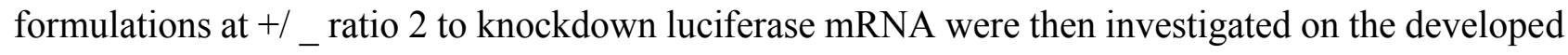
metastatic model of human lung A549 non-small cell lung carcinoma. Mice were randomized in 5 groups and treated once a day for 3 days by intravenous injections of either $\mathrm{NaCl} 0.9 \%$, luciferasesiRNA solution, HA-lipoplexes prepared with nonspecific control siRNA, HA-lipoplexes prepared with luciferase siRNA or non-modified lipoplexes prepared with luciferase siRNA. Tumor growth, calculated as the bioluminescence on the thoracic region after treatment relative to the bioluminescence before treatment, is shown in Fig. 5. Treatment with HA-lipoplexes carrying luc siRNA led to a statistically significant decrease of luciferase expression in Fig. 2. Uptake kinetics of rhodamine-labeled non-modified (blue) and HA-modified (red) liposomes and lipoplexes by A549luc cells, quantified by flow cytometry as the mean fluorescence intensity (MFI). Cells were incubated for $48 \mathrm{~h}$ with particles at a lipid concentration of $10 \mathrm{mM}(\mathrm{n}=3)$. (For interpretation of the references to colour in this figure legend, the reader is referred to the web version of this article.). Fig. 3. Luciferase expression inhibition in A549-luc cells by siRNA lipoplexes at + / ratios 134 and 2 prepared with non-modified or HA-liposomes. Cells were incubated with lipoplexes for $48 \mathrm{~h}$ before luminescence measurements $(\mathrm{n}=8)$ comparison with controls and non-modified lipoplexes. While tumor luminescence increased to $102 \ldots 13 \%$ after treatment with vehicle and to $133 \ldots 27 \%$ after treatment with non-modified lipoplexes, it decreased to $83 \ldots 27 \%$ after treatment with HA-lipoplexes.

\subsection{Quantification of luciferase mRNA expression by RT-qPCR}


The objective of the real-time PCR assays was to determine if the observed decrease in tumor luminescence could be identified as the inhibition of luciferase mRNA expression in the lung tissues. The transcriptional expression analysis showed a 54\% reduction of the expression of PGL3-luc transcript in the lung of animals treated with HA-lipoplexes compared to animals treated with $\mathrm{NaCl}$ $0.9 \%$ (Fig. 6). Combined with the results from the luminescence measurements, it confirms the inhibitory activity of siRNA lipoplexes upon the expression of PGL3-luc in our model.

\section{Discussion}

The aim of this study was to characterize in vitro and in vivo the potential of HA-modified lipoplexes for the targeted delivery of siRNA to CD44-expressing A549 lung cancer cells. As we have previously observed that this amount was optimal for the transfection of MDA-MB231 and A549 cancer cells expressing CD44 (Dufay Wojcicki et al., 2012; Surace et al., 2009), 10\% (wHA/ wtotal lipids) of HA-DOPE conjugate (Surace et al., 2009) was used to prepare HA-modified lipoplexes, namely HA-lipoplexes, in which the DOPE lipid served as an anchor for the insertion of HA on lipid bilayer. Lipoplexes at +/_ ratio 2 were used for the experiments, and lipoplexes at +/ _ratio 134 were introduced in some cases as a control of positively charged lipoplexes. Indeed in a Fig. 4. Representative image of the lipoplexes distribution in the lungs of mice. Pictures were taken with a $10 \mathrm{x}$ magnification. From left to right, first frame: image taken using the filter for DAPI staining (cells), second frame: image taken using the filter for rhodamine staining (lipoplexes), third frame: merge, showing lungs cells in blue and lipoplexes in red. Non-modified lipoplexes $+/$ _ ratio 2 and HA-lipoplexes +/_ ratio 2 were administered by intravenous injection. Animals were euthanized and lungs were dissected and flash-frozen $30 \mathrm{~min}$ after treatment. Scale bars $=100 \mathrm{~mm}$. (For interpretation of the references to colour in this figure legend, the reader is referred to the web version of this article.) Fig. 5. Luciferase expression inhibition in nude athymic mice, calculated as the bioluminescence on the thoracic region after treatment compared to the biolumines- cence before treatment with lipoplexes at $+/$ _ ratio 2 . Mice were inoculated intravenously with 1.5 millionA549luccells. After 4weeks, animals were treated once a day for 3 days by intravenous injections of $\mathrm{NaCl}$ $0.9 \%$, luciferase-siRNA solution, HA-lipoplexes prepared with nonspecific control siRNA, HAlipoplexes prepared with luciferase siRNA or non-modified lipoplexes prepared with luciferase siRNA. Data represent the mean _ SD ( $\ldots$ 6). ${ }^{*}, \mathrm{P}<0.05$ versus vehicle. $108 \mathrm{~T}$. Leite Nascimento et al. / International Journal of Pharmaceutics 514 (2016) 103-111recent publication, we have shown that for this specific ratios, the charge was different as well as the morphology of the particles (Nascimento et al., 2015). Moreover in lipoplexes at + _ $_{\text {_ }}$ ratio 2 only $36 \%$ of the HA was associated to particles compared to $79 \%$ for lipoplexes at +/_ ratio 134 (Nascimento et al., 2015) and we assume 
this could affect cellular interactions. Lipoplexes showed increased diameter after insertion of the DOPE-HA conjugate and the addition of siRNA, reaching $230 \mathrm{~nm}$ for the HA-lipoplexes at +/ _ ratio 2, that presented the highest amounts of associated siRNA. This effect was already described for HAmodified liposomes (Arpicco et al., 2013a; Gan et al., 2013; Tiantian et al., 2014), since HA is a hydrophilic polymer and its placement on membrane surface increases particle hydrodynamic diameter. The multilamellar characteristic of the lipoplexes revealed by cryomicroscopy in a previous study (Nascimento et al., 2015), permitted a siRNA entrapment efficacy of $96.0 \ldots 2.6 \%$ that were located between bilayers as shown in this earlier report (Nascimento et al., 2015). HA lipoplexes were more stable than their counterparts with no HA due to a stabilization mechanism being the repulsion between negative charges of HA and serum proteins, minimizing their nonspecific interactions and causing particle stabilization. The biological activity of HA-lipoplexes was evaluated using the A549luc human lung carcinoma cell line, which proved to be a suitable model for CD44 targeting since the totality of the cell population exhibited the CD44 membrane receptor. Measurement of cellular mitochondrial activity of cells exposed to liposomes/ lipoplexes showed that the modification by HA did not change lipoplex toxicity. This biocompatibility is consistent with the ubiquitous and nontoxic characteristics of HA (Arpicco et al., 2013a), and is in agreement with previous studies in which the modification of neutral (Glucksam-Galnoy et al., 2012; Peer and Margalit, 2004a), negatively (Arpicco et al., 2013b; Mizrahy et al., 2011) or positively charged (Surace et al., 2009) liposomes with HA revealed no apparent effect on cellular proliferation of CD44- overexpressing cells. The cytotoxicity of cationic lipids and polymers has been investigated in several studies, especially due to the increasing interest in these compounds for their ability of forming lipoplexes upon incubation with negatively charged nucleic acids (Lv et al., 2006; Morille et al., 2008; Zhang et al., 2004). When present on nanoparticles, these cationic compounds, upon binding to the cell surface, can be recognized as a signal of danger for cells (inducing pro-apoptotic or pro-inflammatory reactions) or contribute to activate cascades that are classically activated by endogenous cationic compounds (Lonez et al., 2012). This toxicity depends though on several factors besides the particle surface charge, such as the lipid type, amount and charge (Park et al., 2014), and its ratio in combination with helper lipids (Morille et al., 2008). Our results suggest that the cytotoxicity profile of HA-lipoplexes is not related to the changes in particle surface charge, since cells responded similarly when incubated with all formu-lations, and that cytotoxicity was observed only after incubation with high concentration of particles. Quantification of lipid-related fluorescence by flow cytometry showed higher internalization in A549 CD44-overexpressing cells of samples that contained the conjugate, confirming the crucial role of the receptor in the endocytosis process, as shown previously by our group and others (Dalla Pozza et al., 2013; Dufay Wojcicki et al., 2012; Ganesh et al., 2013; Mizrahy 
et al., 2011; Qhattal and Liu, 2011; Taetz et al., 2009). Despite that the higher fluorescence Fig. 6. Relative expression of PGL3-LUC mRNA transcript in nude athymic mice, calculated using r18S, HPRT, TBP and ACTB genes as references for normalization of mRNA expression. Results are expressed as the $\mathrm{n}$-fold differences in target gene expression in HA-lipoplex-treated vs $\mathrm{NaCl} 0.9 \%$ treated mice. Data represent the mean _ SD $(n=3 ; 4)$. T. Leite Nascimento et al. / International Journal of Pharmaceutics 514 (2016) 103-111 109associated to the uptake of the plain lipoplexes +/ - ratio 2 at $48 \mathrm{~h}$ masked the effect of HA presence on the internalization of these lipoplexes, cell fluorescence intensity was clearly higher for HA-lipoplexes in all other time points. These data correlated with the ability of anti-Luc siRNA lipoplexes in promoting more than $80 \%$ luciferase inhibition with HA-lipoplexes at + / $_{\text {_ ratio }} 2(10 \%$ more than non-modified lipoplexes at the same ratios) and $65 \%$ with HA-DOPE lipoplexes at $+/$ _ ratio 134 . This corresponds to $67 \mathrm{nM}$ and $1 \mathrm{nM}$ siRNA/well, respectively and demonstrates the high siRNA delivery efficiency of the lipoplexes. This is in agreement with the lipoplexes uptake studies showing that the overall amount of lipoplexes being taken up by cells at $+/{ }_{-} 2$ was similar. The preferential uptake and thus gene expression inhibition effect of the HA-lipoplexes + / $_{\text {_ }}$ ratio 2 is masked by the continuous internalization of the nonmodified lipoplexes +/_ratio 2. Despite these similarities, these latest formulation was further chosen for in vivo experiments since the HA-DOPE lipoplexes at + / _ ratio 134 carried insufficient amount of siRNA to obtain an inhibition effect in the animal model (data not shown). The modification of nanoparticles with HA has been explored mainly for its ability to specifically bind to various cells that overexpress CD44, a common marker for tumor initiating cells-cancer stem cells (CSC) in human carcinomas. Since a variety of cells, including breast, ovarian, colon, stomach, and lung carcino-mas (Day and Prestwich, 2002) overexpress these receptors, the formulation of HA-modified particles is a promising strategy to promote receptor-mediated cellular entry. This strategy has been proved largely efficient in vitro (Arpicco et al., 2013b). When in vivo activity is tested, though, results are less outstanding. Most of the currently developed nanosystems, including the actively targeted ones, exhibit a discrepancy between targetability in vitro and in vivo (Jiang et al., 2012). Long blood circulation is critical for active tumor targeting, and to reach the targeted cells after intravenous injection, nanoparticles first have to resist clearance from the circulation by the monocyte phagocytic system. This is not the case for all HA-modified nanoparticles. Even though HA has been successfully used as a hydrophilic coating and even proposed as an alternative to PEG to increase blood circulation of nanoparticles (Jiang et al., 2012; Mizrahy et al., 2011; Peer and Margalit, 2004a; b). Qhattal et al. (Qhattal et al., 2014) exemplified that depending on the polymer length of HA and on their negative surface charge, HA-liposomes may even suffer a faster clearance. Therefore, a case-by-case analysis of the in vivo fate of HA-particles is necessary. Tissue analysis after administration of rhodamine- 
labeled HA-lipoplexes in healthy CF-1 mice revealed diffuse fluorescence corresponding to the HAlipoplexes in the mouse lung, which was not the case with non-modified lipoplexes which formed aggregates. These data correlates well with the low stability of plain lipoplexes compared to the ones covered by HA. To test the hypothesis that HA-lipoplexes can transport siRNA into the cytoplasm of CD44-overexpressing cells in vivo, we developed a model of human lung A549 non-small cell lung carcinoma. The bioluminescence images and appearance of lung nodules con-firmed lung metastasis. Lipoplexes were then administered by bolus intravenous injection, at a volume of $0.1 \mathrm{~mL}$ per $10 \mathrm{~g}$ of body weight, which is normally acceptable in human therapy. Results confirmed the advantage of HA-lipoplexes over non-modified lipoplexes on the inhibition of luciferase expression by decrease in luminescence and PGL3-LUC transcript in the tumors. Taken together, these results indicate that our HA-lipoplexes are able to carry the siRNA molecules to the cytoplasm of CD44-overexpress- ing cells. These HA-modified carriers beneficiate of the EPR effect, as consequence of the increase in hydrophilicity and longer circulation time caused by the presence of HA on the surface of the particles, and of the CD44-targeting promoted by HA moieties (Park et al., 2014). The results presented here show the potentialities of HA-lipoplexes as siRNA carriers to CD44-overexpressing cells. Lipoplexes are stable in the presence of serum, and their structural modification with the HADOPE conjugate improves cellular internalization mediated by the CD44 receptor. After uptake, lipoplexes are distributed in the cytoplasm, where the carried siRNA promotes highly efficient gene expression inhibition. The HA-lipoplexes are able to transport in vivo a dose of intact siRNA for uptake and ultimately intracellular function, and are thus a promising tool for anticancer therapeutic gene expression inhibi-tion.

\section{Acknowledgments}

This work was financially supported by CAPES, Brazil (process number 5641-10-1). 


\section{References}

Aagaard, L., Rossi, J.J., 2007. RNAi therapeutics: principles, prospects and challenges. Adv. Drug Deliv. Rev. 59, 75-86.

Al-Hajj, M., Wicha, M.S., Benito-Hernandez, A., Morrison, S.J., Clarke, M.F., 2003. Prospective identification of tumorigenic breast cancer cells. Proc Natl Acad Sci U. S. A. 100, 3983-3988. Alshaer, W., Hillaireau, H., Vergnaud, J., Ismail, S., Fattal, E., 2015. Functionalizing liposomes with anti-CD44 aptamer for selective targeting of cancer cells. Bioconjug. Chem. 26, 1307-1313.

Arpicco, S., Canevari, S., Ceruti, M., Galmozzi, E., Rocco, F., Cattel, L., 2004. Synthesis, characterization and transfection activity of new saturated and unsaturated cationic lipids. Farmaco $59,869-878$.

Arpicco, S., De Rosa, G., Fattal, E., 2013a. Lipid-based nanovectors for targeting of CD44overexpressing tumor cells. J. Drug Deliv. 2013.

Arpicco, S., Lerda, C., Dalla Pozza, E., Costanzo, C., Tsapis, N., Stella, B., Donadelli, M., Dando, I., Fattal, E., Cattel, L., Palmieri, M., 2013b. Hyaluronic acid-coated liposomes for active targeting of gemcitabine. Eur. J. Pharm. Biopharm. 85, 373-_380.

Batzri, S., Korn, E.D., 1973. Single bilayer liposomes prepared without sonication. Biochim. Biophys. Acta 298, 1015-1019.

Dalla Pozza, E., Lerda, C., Costanzo, C., Donadelli, M., Dando, I., Zoratti, E., Scupoli, M. T., Beghelli, S., Scarpa, A., Fattal, E., Arpicco, S., Palmieri, M., 2013. Targeting gemcitabine containing liposomes to CD44 expressing pancreatic adenocarcinoma cells causes an increase in the antitumoral activity. Biochim. Biophys. Acta 1828, 1396-1404.

Day, A.J., Prestwich, G.D., 2002. Hyaluronan-binding proteins: tying up the giant. J. Biol. Chem. $277,4585-4588$.

De Rosa, G., De Stefano, D., Laguardia, V., Arpicco, S., Simeon, V., Carnuccio, R., Fattal, E., 2008. Novel cationic liposome formulation for the delivery of an oligonucleotide decoy to NF-kappaB into activated macrophages. Eur. J. Pharm. Biopharm. 70, 7-18.

Deed, R., Rooney, P., Kumar, P., Norton, J.D., Smith, J., Freemont, A.J., Kumar, S., 1997. Earlyresponse gene signalling is induced by angiogenic oligosaccharides of hyaluronan in endothelial cells. Inhibition by non-angiogenic, high-molecular- weight hyaluronan. Int. J. Cancer 71, 251-256.

Devi, G.R., 2006. siRNA-based approaches in cancer therapy. Cancer Gene Ther. 13, 819-829. Dosio, F., Arpicco, S., Stella, B., Fattal, E., 2016. Hyaluronic acid for anticancer drug and nucleic acid delivery. Adv. Drug Deliv. Rev. 97, 204-236.

Dufay Wojcicki, A., Hillaireau, H., Nascimento, T.L., Arpicco, S., Taverna, M., Ribes, S., Bourge, M., Nicolas, V., Bochot, A., Vauthier, C., Tsapis, N., Fattal, E., 2012. Hyaluronic acid-bearing 
lipoplexes: physico-chemical characterization and in vitro targeting of the CD44 receptor. J. Control. Release 162, 545-552.

Dykxhoorn, D.M., Lieberman, J., 2006. Knocking down disease with siRNAs. Cell 126, 231-235. Fattal, E., Barratt, G., 2009. Nanotechnologies and controlled release systems for the delivery of antisense oligonucleotides and small interfering RNA. Br. J. Pharmacol. 157, 179-194.

Fattal, E., Bochot, A., 2008. State of the art and perspectives for the delivery of antisense oligonucleotides and siRNA by polymeric nanocarriers. Int. J. Pharm. 364, 237-248.

Gan, L., Wang, J., Zhao, Y., Chen, D., Zhu, C., Liu, J., Gan, Y., 2013. Hyaluronan-modified coreshell liponanoparticles targeting CD44-positive retinal pigment epithelium cells via intravitreal injection. Biomaterials 34, 5978-5987. 110

Ganesh, S., Iyer, A.K., Morrissey, D.V., Amiji, M.M., 2013. Hyaluronic acid based self-assembling nanosystems for CD44 target mediated siRNA delivery to solid tumors. Biomaterials.

Glucksam-Galnoy, Y., Zor, T., Margalit, R., 2012. Hyaluronan-modified and regular multilamellar liposomes provide sub-cellular targeting to macrophages, without eliciting a pro-inflammatory response. J. Control. Release 160, 388-393.

Hellemans, J., Mortier, G., De Paepe, A., Speleman, F., Vandesompele, J., 2007. qBase relative quantification framework and software for management and automated analysis of real-time quantitative PCR data. Genome Biol. 8, 14.

Jiang, T., Zhang, Z., Zhang, Y., Lv, H., Zhou, J., Li, C., Hou, L., Zhang, Q., 2012. Dual-functional liposomes based on $\mathrm{pH}$-responsive cell-penetrating peptide and hyaluronic acid for tumor-targeted anticancer drug delivery. Biomaterials 33, 9246-9258.

Landesman-Milo, D., Goldsmith, M., Leviatan Ben-Arye, S., Witenberg, B., Brown, E., Leibovitch, S., Azriel, S., Tabak, S., Morad, V., Peer, D., 2013. Hyaluronan grafted lipid-based nanoparticles as RNAi carriers for cancer cells. Cancer Lett. 334, 221-227.

Laurent, T.C., Fraser, J., 1992. Hyaluronan. FASEB J. 6, 2397-2404. Lonez, C., Vandenbranden, M., Ruysschaert, J.-M., 2012. Cationic lipids activate intracellular signaling pathways. Adv. Drug Deliv. Rev. 64, 1749-1758.

Lv, H., Zhang, S., Wang, B., Cui, S., Yan, J., 2006. Toxicity of cationic lipids and cationic polymers in gene delivery. J. Control. Release 114, 100-109. Mizrahy, S., Raz, S.R., Hasgaard, M., Liu, H., Soffer-Tsur, N., Cohen, K., Dvash, R., Landsman-Milo, D., Bremer, M.G.E.G., Moghimi, S.M., 2011. Hyaluronan-coated nanoparticles: the influence of the molecular weight on CD44-hyaluronan interactions and on the immune response. J. Control. Release 156, 231-238.

Morille, M., Passirani, C., Vonarbourg, A., Clavreul, A., Benoit, J.-P., 2008. Progress in developing cationic vectors for non-viral systemic gene therapy against cancer. Biomaterials 29, 3477-3496. 
Nascimento, T.L., Hillaireau, H., Fattal, E., 2012. Nanoscale particles for lung delivery of siRNA. J. Drug Deliv. Sci. Tec. 22, 99-108.

Nascimento, T.L., Hillaireau, H., Noiray, M., Bourgaux, C., Arpicco, S., Pehau- Arnaudet, G., Tavema, M., Cosco, D., Tsapis, N., Fattal, E., 2015. Supramolecular organization and siRNA binding of hyaluronic acid-coated lipoplexes for targeted delivery to the CD44 receptor. Langmuir 31, 1118611194.

Noble, P.W., 2002. Hyaluronan and its catabolic products in tissue injury and repair. Matrix Biol. 21, 25-29. Park, J.-H., Cho, H.-J., Yoon, H.Y., Yoon, I.-S., Ko, S.-H., Shim, J.-S., Cho, J.-H., Park, J.H., Kim, K., Kwon, I.C., 2014. Hyaluronic acid derivative-coated nanohybrid liposomes for cancer imaging and drug delivery. J. Control. Release 174, 98-108.

Peer, D., Margalit, R., 2004a. Loading mitomycin C inside long circulating hyaluronan targeted nanoliposomes increases its antitumor activity in three mice tumor models. Int. J. Cancer 108, 780-789. Peer, D., Margalit, R., 2004b. Tumor-targeted hyaluronan nanoliposomes increase the antitumor activity of liposomal Doxorubicin in syngeneic and human xenograft mouse tumor models. Neoplasia $6,343-353$.

Peer, D., Florentin, A., Margalit, R., 2003. Hyaluronan is a key component in cryoprotection and formulation of targeted unilamellar liposomes. Biochim. Biophys. Acta 1612, 76-82.

Qhattal, H.S.S., Liu, X.L., 2011. Characterization of CD44-mediated cancer cell uptake and intracellular distribution of hyaluronan-grafted liposomes. Mol. Pharm. 8, 1233-1246.

Qhattal, H.S.S., Hye, T., Alali, A., Liu, X., 2014. Hyaluronan polymer length, grafting density, and surface poly(ethylene glycol) coating influence in vivo circulation and tumor targeting of hyaluronangrafted liposomes. ACS Nano 8, 5423-5440.

Rivkin, I., Cohen, K., Koffler, J., Melikhov, D., Peer, D., Margalit, R., 2010. Paclitaxel- clusters coated with hyaluronan as selective tumor-targeted nanovectors. Biomaterials 31, 7106-7114. Ruhela, D., Kivimäe, S., Szoka, F.C., 2014. Chemoenzymatic synthesis of oligohyaluronan-lipid conjugates. Bioconjug. Chem. 25, 718-723.

Soutschek, J., Akinc, A., Bramlage, B., Charisse, K., Constien, R., Donoghue, M., Elbashir, S., Geick, A., Hadwiger, P., Harborth, J., John, M., Kesavan, V., Lavine, G., Pandey, R.K., Racie, T., Rajeev, K.G., Rohl, I., Toudjarska, I., Wang, G., Wuschko, S., Bumcrot, D., Koteliansky, V., Limmer, S., Manoharan, M., Vornlocher, H.P., 2004. Therapeutic silencing of an endogenous gene by systemic administration of modified siRNAs. Nature 432, 173-178.

Surace, C., Arpicco, S., Dufay-Wojcicki, A., Marsaud, V., Bouclier, C., Clay, D., Cattel, L., Renoir, J.M., Fattal, E., 2009. Lipoplexes targeting the CD44 hyaluronic acid receptor for efficient transfection of Breast cancer cells. Mol. Pharm. 6, 1062-_ 1073. 
Taetz, S., Bochot, A., Surace, C., Arpicco, S., Renoir, J.M., Schaefer, U.F., Marsaud, V., KerdineRoemer, S., Lehr, C.M., Fattal, E., 2009. Hyaluronic acid-Modified DOTAP/DOPE liposomes for the targeted delivery of anti-Telomerase siRNA to CD44-expressing lung cancer cells. Oligonucleotides 19, 103-115.

Tiantian, Y., Wenji, Z., Mingshuang, S., Rui, Y., Shuangshuang, S., Yuling, M., Jianhua, Y., Xinggang, Y., Shujun, W., Weisan, P., 2014. Study on intralymphatic-targeted hyaluronic acidmodified nanoliposome: influence of formulation factors on the lymphatic targeting. Int. J. Pharm. 471, 245-257.

Vandesompele, J., De Preter, K., Pattyn, F., Poppe, B., Van Roy, N., De Paepe, A., Speleman, F., 2002. Accurate normalization of real-time quantitative RT-PCR data by geometric averaging of multiple internal control genes. Genome Biol. 3, 12.

Wang, L., Su, W., Liu, Z., Zhou, M., Chen, S., Chen, Y., Lu, D., Liu, Y., Fan, Y., Zheng, Y., Han, Z., Kong, D., Wu, J.C., Xiang, R., Li, Z., 2012. CD44 antibody-targeted liposomal nanoparticles for molecular imaging and therapy of hepatocellular carcinoma. Biomaterials 33, 5107-5114.

Yang, X.-Y., Li, Y.-X., Li, M., Zhang, L., Feng, L.-X., Zhang, N., 2013. Hyaluronic acid-coated nanostructured lipid carriers for targeting paclitaxel to cancer. Cancer Lett. 334, 338-345.

Zhang, S., Xu, Y., Wang, B., Qiao, W., Liu, D., Li, Z., 2004. Cationic compounds used in lipoplexes and polyplexes for gene delivery. J. Control. Release 100, 165-180. 